\title{
Impacto mediático de los fenómenos medioam- bientales: la sequía en el sur de España durante el último medio siglo y su cobertura en prensa'
}

\author{
Teodoro LEÓN GROSS \\ Universidad de Málaga \\ teo@uma.es \\ José Damián RuIz SinogA \\ Universidad de Málaga \\ sinoga@uma.es
}

Recibido 12 de marzo de 2012

Aceptado: 28 de septiembre de 2012

\begin{abstract}
Resumen
Las situaciones climáticas extremas se han acentuado en el Mediterráneo durante el siglo XX -como su proyección a través de los medios de comunicación- por lo que su análisis constituye una línea prioritaria para llevar a cabo estrategias de planificación y mitigar sus efectos. La sequía es uno de los fenómenos de consecuencias más nefastas y el más complejo al ser de aparición lenta, sin que se reconozca hasta que las actividades humanas y el medio ambiente ya están afectados, con efectos que pueden persistir largo tiempo. Este estudio interdisciplinar evalúa la existencia de una cobertura periodística solvente del fenómeno.
\end{abstract}

Palabras clave: Prensa, cobertura periodística, medio ambiente, cambio climático, sequía.

\section{Media Impact of Environmental Phenomena: South Spain drought along the last half-century and its press coverage}

\begin{abstract}
Extreme climate situations were accentuated in the Mediterranean areas during the 20th century, as its projection through the communication media. Their analysis constitutes a priority topic to carry out planning strategies and mitigate their effects. The drought is one of the most disastrous consequences phenomena and the most complex due to her slow onset, without that recognize until that human activities and the environment are already affected, with effects that can persist for a long time. This interdisciplinary study assesses the existence of a solvent media coverage of the phenomenon.
\end{abstract}

Keywords: Press, Journalistic Coverage, Environment, Climate Change, Drouhgt.

Referencia normalizada: LEÓN GROSS, Teodoro y RUIZ SINOGA, José Damián (2012): “Impacto mediático de los fenómenos medioambientales: la sequía en el sur de España durante el último medio siglo y su cobertura en prensa". Estudios sobre el mensaje periodístico, vol. 18, núm. 2 (julio-diciembre), págs.: 769-790. Madrid, Servicio de Publicaciones de la Universidad Complutense.

Sumario: 1. Introducción. 2. Metodología; 2.1. Utilización del SPI; 2.2. Aproximación al grado de sensibilidad social: análisis de contenido mediático. 3. Área de estudio. 4. Resultados y discusión; 4.1. Tipología de las sequías; 4.2. Las rachas secas; 4.3. Las sequías hidrológicas; 4.4. Percepción social de las sequías desde la agenda massmediática; 4.4.1. Volumen; 4.4.2. Tamaño; 4.4.3. Género; 4.4.4. Fuentes; 4.4.5. Tono; 4.4.6. Temas. 5. Conclusiones. 6. Referencias bibliográficas.

1 El presente estudio se ha realizado con la financiación de los Proyectos CGL2010-21425C02-01 y CSO2010-19725 dentro del Plan Nacional de Investigación del entonces Ministerio de Ciencia e Innovación, convocatoria de 2010. 


\section{Introducción}

El tratamiento mediático es un plano esencial para el entendimiento de la realidad. Cualquier fenómeno social es indisociable de la construcción materializada en los medios de comunicación masiva. En definitiva, "lo que sabemos de nuestra sociedad, incluso acerca del mundo en que vivimos, lo sabemos por los medios de comunicación. Esto no vale únicamente para nuestro conocimiento de la sociedad y la historia, sino para nuestro conocimiento de la naturaleza" (LuHMANN, 1996:9). Y si es un hecho que la mayor parte del conocimiento está determinado por la acción de los mediadores; desde hace décadas se ha anotado que el público adquiere casi la totalidad de su conocimiento sobre asuntos científicos a través de los medios de comunicación (así NELKIN, 1987). Así pues, las representaciones mediáticas son un factor determinante en el entendimiento y compromiso público ante la ciencia climática y por tanto merecen una consideración obvia (BOYKOFF 2008); y es algo que se ha enfatizado particularmente respecto al denominado 'cambio climático', insistiendo en la relación muy directa en el tratamiento mediático y la opinión pública (PETERs y HeINRICHS, 2005). Esto es esencial porque se traslada un conocimiento complejo a la lógica de los medios; algo que a menudo provoca distorsiones de modo que la traducción de asuntos científicos al lenguaje periodístico más o menos popular acaba dando lugar a desconfianza o incluso acusaciones de sensacionalismo (LADLE, 2004). Hay un amplio desarrollo de investigaciones en torno a la agenda-setting function y sus efectos, sobre la tendencia a crear frames enmarcando la percepción de la realidad (ENTMAN, 1993) y eliminando las realidades poliédricas para fijar el punto de vista de los mass media; porque además la persuasión de esos enfoques aumenta cuanto mayor es la exposición del público a tales enfoques. En el ámbito mediterráneo las situaciones climáticas extremas se han incrementado durante el siglo XX, y dentro de estas anomalías climáticas, las sequías son las de consecuencias más nefastas y las más complejas de todas ellas (WILHITE, 1993). Mientras que otros riesgos climáticos como las inundaciones, tienen una mayor concreción espacio-temporal, en el caso de las sequías resulta complejo determinar su inicio y final, y normalmente afectan a áreas más amplias (CUADRAT Prats y Vicente-SerRANo, 2005). La aparición de la sequía suele ser lenta y, a menudo, no se reconoce hasta que las actividades humanas o el medio ambiente quedan afectados por ella; e incluso sus efectos pueden persistir mucho tiempo después de que haya terminado (CHANGNON \& EASTERLING, 1989); de modo que la respuesta mediática, que carece de la concreción de la inmediatez, resulta reveladora.

Las sequías en el sur de España constituyen un fenómeno de dimensión estructural que afecta a toda la vida en el territorio, con una fuerte repercusión socioeconómica, por lo que su análisis, tipología y caracterización es una línea prioritaria al objeto de llevar a cabo estrategias políticas de planificación (EASTERLING et al, 2000; KUNKEL et al., 1999) puesto que además, como señala MoraLES et al., (2000), en áreas litorales se ha producido un incremento de las demandas hídricas por parte de las actividades turísticas y en algunas áreas de la agricultura de regadío. La sequía adquiere por tanto, junto a su carácter relacionado con el aprovisionamiento de los recursos hídricos, un aspecto socioeconómico y ambiental, relacionado también con indicadores de calidad o confortabilidad climática. Esto confiere valor a la iniciativa 
de sondear la calidad de la cobertura periodística, sobre todo al disponer de una serie histórica de mediciones de absoluta fiabilidad.

En el presente estudio, se analiza la cobertura mediática a partir del calendario de anomalías pluviométricas estudiadas para los últimos 50 años (Standarized Precipitation Index) en 4 observatorios representativos de la Cuenca Sur de España, dos de ellos localizados en el litoral (Málaga y Algarrobo) y otros dos en cabeceras de cuencas reguladas (ANTEQUERA y PERIANA), con los objetivos citados de realizar un análisis tipológico de las sequias, frecuencia e intensidad, para establecer asimismo la dinámica y evolución de la percepción social materializada a través de los medios de comunicación en el contexto del cambio global.

El conocimiento climático se convierte en un factor de alta rentabilidad social que requiere un estado de conciencia colectiva que implica a los medios de comunicación, agentes integradores de coherencia social al construir la realidad de referencia. Los propios ciudadanos tienen diferentes expectativas en relación a los medios que, más allá de la información de entretenimiento, incluye la provisión de información útil que ayuda a la gente en su vida, y la provisión de información fiable que aporta seguridad ontológica en un mundo inseguro. Las noticias, bajo esta premisa, deben evaluarse en el grado en que se responde alguna o todas estas expectativas (COLEMAN et al., 2009).

\section{Metodología}

El análisis de contenido es el método utilizado para medir la respuesta mediática ante el fenómeno de la sequía. Se trata de lograr "la emergencia de aquel sentido latente que procede de las prácticas sociales y cognitivas que instrumentalmente recurren a la comunicación para facilitar la interacción que subyace a los actos comunicativos concretos y subtiende la superficie material del texto" (PIÑUEL, 2002:4). Este se ha manifestado como un método competente para la des-ocultación o re-velación de lo latente o potencial del mensaje; pero además no sólo permite inferencias a partir del texto sino que, hacia la dimensión no manifiesta del texto, asumida su complejidad, facilita una combinación eficaz con otras variables en el análisis a fin de que el texto cobre el sentido requerido para el analista. En este estudio se trabaja complementariamente con el SPI para el análisis de las sequías. Ciertamente existen múltiples métodos ya clásicos para analizar la intensidad, frecuencia y duración de las anomalías secas, como el BMDl (Bhalme y Mooley, 1980), el análisis de periodos de retorno móviles (Perez Cueva y Escrivá, 1982), el RAl (Rooy. 1965); el índice de Gibbs (1975); el índice de Thonhwaite (1948), el índice de Palmer (PDSI, PALMER, 1965), etcétera; sin embargo, de los diferentes indicadores, el mas utilizado ha sido el Standardized Precipitation Index (SPI), desarrollado por MCKEE et al. (1993 y 1995) para identificar los periodos secos y húmedos de modo más preciso. KEYANTASH \& DRACUP (2002) analizaron la consistencia de 18 índices de sequía y concluyeron que el SPI es el que mejor tipifica las sequías, las compara espacialmente y cuantifica la severidad, duración y magnitud espacial de las mismas. Aunque el desarrollo del SPI es reciente, se está utilizando ampliamente para el estudio de las sequías en EEUU (HAYES et al., 1999), en Europa (Lloyd-Hughes \& SAunders, 2002) y en la Peninsula Iberica (Poquet et al, 2008a; Cuadrat Prats y Vicente-Serrano, 2005; etc...). 
Así pues, la investigación se despliega en dos planos para confirmar la hipótesis sobre la calidad de la cobertura periodística ante este fenómeno climático:

- Utilización del SPI

- Análisis de contenido

\subsection{Utilización del SPI}

El Standarized Precipitation Index (SPI) fue desarrollado por McKeE et al., (1993 y 1995) para conseguir una representación de los periodos anormalmente secos y húmedos de forma precisa.

En general, los trabajos desarrollados hasta el momento muestran las ventajas que presenta el SPI en el reconocimiento de los periodos secos (GUTTMAN, 1998). Existen diferentes trabajos que analizan la utilidad del SPI en el seguimiento de las sequías (EDWARDS y MCKeE, 1997; MCKeE et al.. 2000; HAYES et al.. 1999; Wu el al.. 2001; Vicente-SERrano et al., 2004; Vicente-SERrano y LóPEZ-Moreno., 2005; Moreira et al., 2008; LANA et al., 2009).

La información básica necesaria para calcular el SPI debe ser una serie homogénea de precipitaciones mensuales de al menos 30 años de duración (Wu el al., 2001), aunque en este trabajo se ha seguido el criterio de GUTTMAN (1999), que señala la necesidad de disponer de, al menos, 50 años de registros de precipitaciones, previa homogeneización de los datos.

Las sequias en ambientes mediterráneos poseen una variable frecuencia a diferentes escalas temporales de análisis (KomUSCU, 1999). Mientras que a escalas temporales largas (p.ej.: 48 meses) los periodos secos son menos frecuentes pero más duraderos, a escalas cortas ( 3 meses) la frecuencia de estos periodos se incrementa, pero su duración desciende. El SPI a cortas escalas temporales responde rápidamente a un mes muy seco o muy húmedo, lo que significa que cada nuevo mes tiene una larga influencia en el periodo en el que se suma la precipitación. No obstante, cuando la escala temporal se incrementa el índice responde lentamente indicando pocos fenómenos de sequia pero de larga duración. Así pues, considerando que una de las ventajas del SPI es la de tipificar los periodos secos con un amplio espectro de escalas temporales (EDWARDS y MCKEE, 1997), se ha realizado un análisis del mismo a escalas de 3, 6, 12, 24 y 48 meses, al objeto de poder determinar desde las estaciones secas, a las sequías estructurales.

El SPI a una escala de 3 meses es un buen indicador de las sequías estacionales o sequias a corto plazo, mientras que a 12 meses lo es de las sequias de duración intermedia y el SPI de 48 meses se utiliza como un índice de sequia a largo plazo (EDWARDS, 2001). Dependiendo de la duración e intensidad de las sequias, así serán las consecuencias socioeconómicas, ambientales e hidrológicas, de ahí el interés que reviste el uso de diferentes escalas de tiempo. La humedad que contiene el suelo será muy sensible a periodos secos cortos (escalas inferiores a seis meses), lo que supone una menor disponibilidad hídrica para la vegetación, con directas consecuencias ambientales (agostamiento de la biomasa e incremento del riesgo de incendio forestal) y agrícolas (reducción de las cosechas). Pero la determinación de los recursos hídricos disponibles en un área concreta solo podrá realizarse a partir de escalas temporales largas, dado que los embalses actúan como reservorios de acumulación de agua. 
Una vez obtenidos los valores de SPI, McKeE et al., (1993) establecen una categorización con el fin de identificar la severidad de cada uno de los periodos secos identificados. En este estudio hemos determinado la severidad de las sequías en 3 categorías: sequía moderada (SPI -1 to -1.49), sequía severa (-1.50 to -1.99) y sequia extrema ( $\leq$ to -2$)$. Esta clasificación es de enorme utilidad para determinar qué espacios están sufriendo sequías de diferente grado a distintas escalas temporales.

\subsection{Aproximación al grado de sensibilidad social: análisis de contenido mediático} La determinación de la sensibilidad social, al no disponer de series históricas de análisis sociométricos de la realidad, se ha explorado a través de la perspectiva de los medios de comunicación, de modo que el análisis de contenido se antoja la solución más competente para investigaciones de este tipo, toda vez que, como ya apuntaba KRIPPENDORFF (2002), permite hacer inferencias reproducibles y válidas de los datos sobre el contexto de los mismos, o según Laurence BARDIN (1996), explotar indicadores válidos y objetivos para inferir conocimiento. La muestra analizada abarca un calendario de largo recorrido, paralelo al calendario establecido en el SPI, y esto refuerza la elección como herramienta óptima para recopilar, procesar y evaluar grandes cantidades de información (SÁNCHEZ ARANDA, 2005), así como en la descripción de los componentes de los mensajes mediáticos (IGARTUA, 2006).

Con este objetivo, el análisis ha tomado como referente mediático el diario Sur, en función de dos criterios objetivos: se trata del único medio con una continuidad en el mercado durante el periodo objeto de estudio, de modo que la estabilidad del análisis secuencial comparativo no se expone a alteraciones, pero además la cabecera creada en 1937 ha sido el periódico líder en el territorio durante estas décadas, manteniéndose como cabecera dominante (BLANCO CASTILLA, 2005) hasta acaparar el cincuenta por ciento del flujo informativo en su ámbito de difusión con un dominio constatado, de forma polietápica, en los informes de la comunicación social en España publicados desde hace más de dos décadas por diversas entidades (Fundesco, Zeta, APM o Telefónica). Esta función de medio dominante le proporciona además un valor referencial para la inducción rectora de la opinión pública (DíAZ NosTY, 1995), plano relevante en este estudio.

La amplitud de la muestra, con una cobertura en el trabajo de campo que se extiende a lo largo de las cinco décadas en las que aplica el SPI comprendiendo veintisiete años concretos -entre 1996 y 2007- evaluados por ciclos de decenios y por indicadores anuales, aporta una alta fiabilidad a las conclusiones. El espectro de categorías permite inferir análisis válidos:

Volumen. Cómputo del número de textos publicados para evaluar el grado de atención y el relieve en la agenda mediática.

Tamaño. Más allá de la amplitud de la cobertura inferida del campo anterior, permite indagar en la profundidad, deducida no ya del número de trabajos sino de su extensión, para discriminar el diferencial en la construcción de la realidad.

Género. Este indicador permite sondear la complejidad del tratamiento periodístico más allá de su frecuencia y profundidad; y para ello explora la tipología de textos, desde la noticia básica que se limita a la descripción enunciativa de unos pocos datos 
al 'deep reporting' o los artículos de analistas que tratan de plantear una superdefinición o una reflexión deliberativa.

Fuentes. El campo de las fuentes constituye una referencia clave para el análisis de las coberturas periodísticas ya que revela, más allá del volumen y la profundidad o la complejidad, también la cualificación de los trabajos. El valor de las fuentes se mide en el doble eje de cantidad/calidad, puesto que la mayor presencia de fuentes da mayor dialéctica, y la mayor calidad cualifica esa dialéctica.

Tono. El campo del tono se sitúa metodológicamente en un plano conflictivo al exigir una apreciación subjetiva, pero es un hecho que las noticias no son textos de laboratorio sino que incluyen "tono y drama" (MCCoMBS, 1992:817) de modo que conviene explorar ese aspecto.

Temática. La sequía adopta un amplio catálogo de temas que modulan con mucha amplitud la percepción de ésta desde la opinión pública, de modo que este análisis es determinante para inferir si se trata de lecturas económicas, ecológicas, infraestructurales, servicios, etcétera.

\section{3. Área de estudio}

El presente estudio se ha llevado a cabo en el sector central de las cordilleras béticas litorales (Provincia de Málaga, Spain). Se ha analizado una serie mensual de precipitaciones (1960-2009) correspondiente a 24 observatorios, 11 situados en el interior, en cuencas hidrográficas reguladas y principales abastecedoras del litoral (Rio Guadalhorce y Rio Vélez), y 13 en el litoral, en un área de intensa urbanización y gran demanda de recursos hídricos, como es la Costa del Sol.

Mediante el uso del software SPSS V15, se realizaron correlaciones múltiples de la precipitación mensual para toda la serie (Pearson) entre todos los observatorios de las cabeceras de cuencas hidrográficas, e igualmente en todos los del litoral. Posteriormente, se determino el observatorio más representativo tanto de cada una de las cuencas reguladas como de las áreas abastecidas del litoral. Las estaciones u observatorios más representativos fueron Antequera (Cuenca alta del Guadalhorce), Málaga (Litoral-cuenca baja del Guadalhorce), Periana (cuenca alta del rio Vélez), y Algarrobo (litoral-cuenca baja del rio Vélez).

Las 4 estaciones seleccionadas presentan unas correlaciones elevadas $(>0,60)$ y positivas respecto a las de su entorno, lo que estadísticamente muestra su buen nivel de representatividad.

\section{Resultados y discusión}

\subsection{Tipología de las sequías}

Los resultados obtenidos, mediante el uso del coeficiente de correlación Rho de Spearman, muestran en todos los casos una buena significación estadística (nivel 0,01 bilateral) y unas correlaciones elevadas. Sin embargo, esta tónica general, puede matizarse.

1. Tanto los observatorios del litoral, como los del interior, en cualquiera de los SPI considerados, tienen un coeficiente Rho de Spearman próximo a 0.8, indicando una buena correlación dentro de la base de datos. 
2. Los coeficientes Rho de la cuenca del rio Vélez (eje Periana-Algarrobo) son más elevados, que los de la cuenca del Guadalhorce (eje Antequera-Málaga), especialmente para los SPI superiores a 24 meses, debido al efecto pantalla que pueden suponer respecto a las masas de aire las sierras sub-béticas.

3. El numero de meses secos/década muestra 3 situaciones. i) Durante la década de los años 60 y 70, en ningún caso rebasa la cifra de 60, con tendencia al incremento para los índices más bajos (SPI3 y SPI6), y a la reducción para los restantes. ii) Durante la década de los años 80 y 90 dicha cifra adquiere valores siempre por encima de 80 . iii) en la última década, el numero de meses se reduce, para todos los índices, pero siempre situándose por encima de 40 , siendo especialmente significativo el relativo a las sequias más duraderas (SPI24) que supera los 80 meses. Esto coincide con lo planteado por otros autores que han investigado situaciones de sequia bajo condiciones mediterráneas (BORDI et al., 2001a, 2001b, LANA et al., 2001, MAHERAS et al., 1999, VICENTE-SERRANO, 2006a, 2006b)

4. El análisis de la duración de las sequias ofrece un doble escenario, i) el número de meses secos incluidos en sequías de escasa duración (inferior a 6 meses), posee un buen ajuste en todos los observatorios. Son las frecuentes rachas secas típicas del clima mediterráneo, que otros autores también han identificado (MAHERAS et al., 1999, Sirdas \& Sen., 2001, Vicente-SerRANO, 2006a, 2006b). ii). Las sequias de mayor duración (SPI24 y SPI48) son aquellas que presentan una más heterogénea distribución. Se trata de sequías hidrológicas, y por consiguiente, las que poseen una mayor incidencia sobre el ecosistema (perdida de biomasa y biodiversidad, degradación de suelos, incremento de procesos de erosión hídrica,...) y los recursos hídricos (reducción de la capacidad de infiltración de los suelos, de la recarga de acuíferos, pérdida de calidad de aguas subterráneas,...), como han analizado MARTínEz MuriLlo y RuIZ Sinoga, (2007) y Ruiz Sinoga y Martínez Murillo, (2009).

5. La evolución del número de periodos secos, o periodos superiores a más de 3 meses secos consecutivos, muestra cómo, i) en la década de los años 80 es en la que se contabiliza un mayor número de periodos secos, ii) en la década de los años 90 los valores permanecen altos, excepto en Málaga, con cifras por encima de 14, iii) en la última década, todos los valores se reducen respecto a los previos, aunque son siempre superiores a los contabilizados en los años 60-70, salvo en Antequera, cuyos valores se aproximan a los de los años 80 (11 periodos secos). Esta variabilidad ha sido también encontrada en ambientes mediterráneos por diversos autores (LANA et al., 2001; Maheras et al., 1999; Vicente-Serrano et al., 2004; Vicente-Serrano y LóPEZ Moreno, 2005; Vicente-SERrano, 2006a y 2006b).

En definitiva, las diferencias observadas nos hacen suponer la existencia de dos tipos de sequías. Aquellas de una duración inferior a 12 meses, más frecuentes, de menor incidencia y que se enmarcarían dentro de la variabilidad característica de las regiones mediterráneas, y a las que podemos denominar rachas secas. Y las de una duración superior a 12 meses, cuyas consecuencias tanto para el equilibrio de los recursos suelo-agua-planta, como para la población, han podido ser mucho más drástica, o sequías hidrológicas. 


\subsection{Las rachas secas}

La escala temporal inferior a los doce meses tiene una importante utilidad en el seguimiento de los déficits hídricos a corto plazo, porque refleja muy bien el estado de las condiciones de humedad de la vegetación, ya que las necesidades hídricas de la misma se producen a escalas temporales cortas.

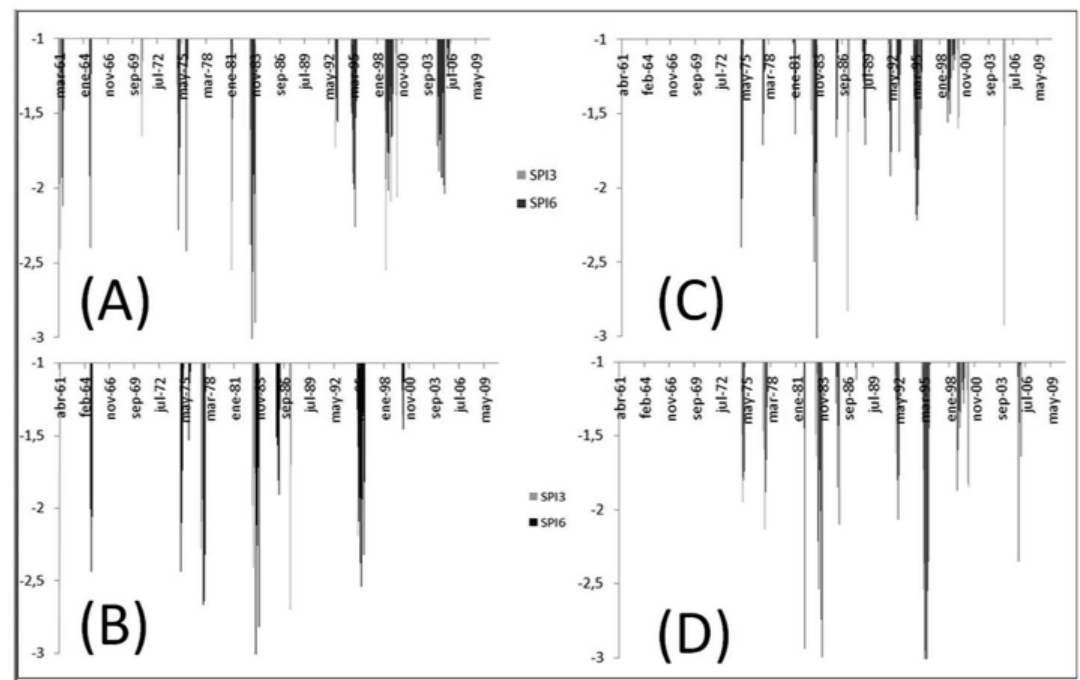

Fig. 1. Rachas secas (1960-2009)

La fig. 1 muestra la evolución temporal de las rachas secas en los observatorios de cabecera de cuenca (arriba) y en los del litoral de su respectiva área tributaria (abajo).

Existe una variabilidad espacial y temporal de la incidencia de estas anomalías pluviométricas, tan características en ecosistemas mediterráneos, cabiendo distinguir entre el eje sub-bético (Antequera-Málaga) con una posible incidencia de las situaciones climáticas de la Cuenca del Guadalquivir y el axarquiense (Periana-Algarrobo) a resguardo de las masas de aire provenientes del norte por la configuración orográfica. Una situación parecida encuentra PoQUET et al., (2008b) en el análisis de los periodos secos en Cataluña.

Además, se constata una cierta coincidencia de las rachas secas entre los observatorios de cabecera y sus homólogos en el litoral, sin embargo, merece ser destacado que:

a) Para todo el periodo considerado, mientras en los años 60 en el sector sub-bético hubo varias rachas secas (1 en Málaga y 3 en Antequera), la primera en el sector axarquiense se constata en el año 1974. En total, se produjeron 9 episodios extremos en Antequera, 6 en Málaga, 4 en Periana y 7 en Algarrobo. Un comportamiento similar ha sido encontrado por Ruiz Sinoga et al., (2010) en el sur de España, por PoQuet et al., (2008a) en Cataluña, y por ViCENTE-SERRANO y LÓPEZ MORENO (2005) en la España interior.

b) Coincidiendo con Vicente-SERRANO (2006a, 2006b), son especialmente significativas por su intensidad las rachas secas correspondientes a los años 198283 y 1994-95, en ambos casos, más acusadas en el litoral que en el interior. 
c) No obstante aparecen episodios que muestran una gran variabilidad en su distribución.

d) Durante la última década, Málaga no padece ninguna racha seca, mientras diferentes episodios de distinta duración (2004-2006 en Antequera, y verano de 2005 en el sector axarquiense) afectaron a las cabeceras de las cuencas abastecedoras, como demuestra RuIZ SinOGA et al., (2010) al analizar la tendencia pluviométrica en el sur de España.

En definitiva, las rachas secas muestran el déficit hídrico a corto plazo, y por tanto permiten conocer las condiciones hídricas del sistema eco-geomorfológico. Se aprecia una frecuente aparición de rachas secas extremas, sobre todo durante las décadas de los años 80 y 90 (MAHERAS et al., 1999; Vicente-SERRANO et al., 2004; VicENTESERRANO y LÓPEZ-MORENO, 2005). En la última década, se contabilizan algunos episodios en las cabeceras y litoral oriental, sin que este tipo de alteraciones afecte al observatorio de Málaga, el área de una mayor aglomeración demográfica.

\subsection{Las sequías hidrológicas}

Para llegar a determinar las sequías hidrológicas es más útil el cálculo del SPI a una escala temporal superior a 12 meses. A esta escala las sequías se hacen menos frecuentes, pero más duraderas, igual que los periodos húmedos. Dado que muestra las condiciones de humedad ya sean anuales o plurianuales, puede servir para el seguimiento de los recursos hídricos a escala de cuenca (SHUKLA \& WOOD, 2008; VicENTESERRANO y CUADRAT, 2002). Se obtienen valores negativos de SPI cuando se produce una larga acumulación de déficits mensuales. Cuando más intenso sea el déficit acumulado más intensa será la sequía y por norma general mayor será su duración (BORDI et al., 2001; PAtel et al., 2007; Vicente-SERRAno y CuAdrat, 2002).

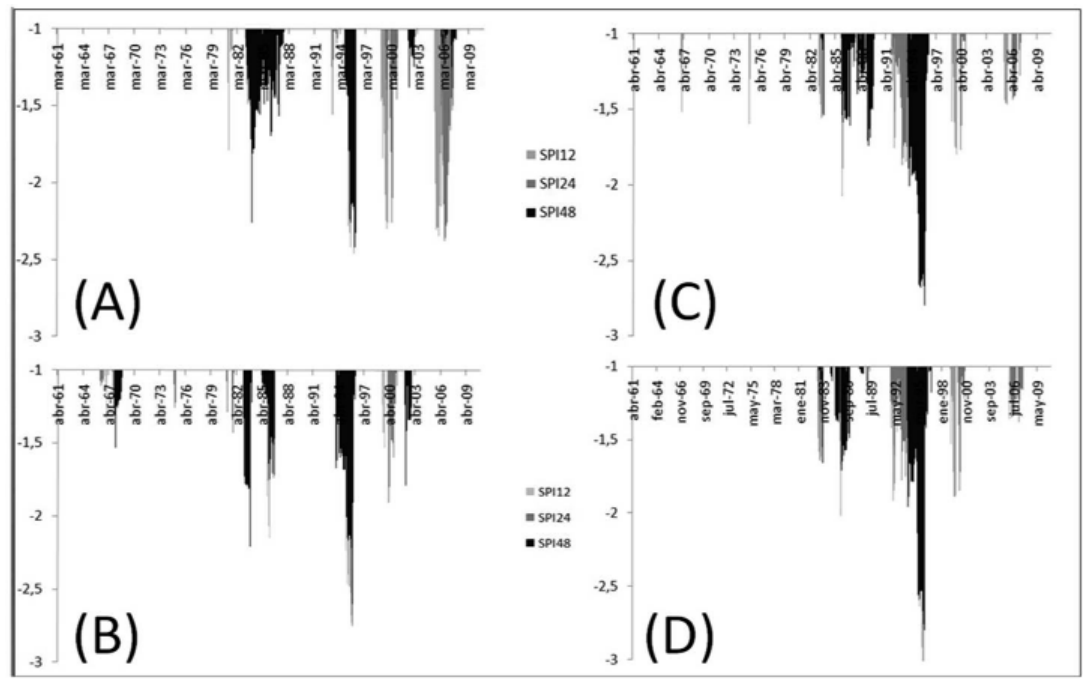

Fig. 2. Sequías Hidrológicas (1960-2009) 
La Fig. 2 muestra la evolución temporal de este tipo de sequías. De su análisis puede observarse como:

a) Las situaciones de sequía estructural adquieren importancia a partir de la década de los 80 .

b) Las sequías tienen una mayor duración en el interior, aquellos que desde el punto de vista hidrológico se corresponden con cuencas reguladas mediante embalses. Similar comportamiento encuentra Lana et al., (2009) en Cataluña.

c) Las sequías tienen una mayor intensidad en los observatorios orientales (Periana y Algarrobo).

d) La reciente sequía (2004-2006), en la zona más poblada (Málaga) no tuvo significación. Fue el observatorio de Antequera, aquel que se ubica en el mayor reservorio de agua, desde el punto de vista hidrológico, el que padeció una sequia estructural de mayor consistencia, debido a su duración cercana a 3 años (Noviembre 2004 a agosto 2007).

e) El numero de meses secos afectados por sequias hidrológicas moderadas es siempre superior al de intensidad severa y extrema. Para el SPI12 estos meses abundan en las décadas de 1980 y 90, sin embargo, en el SPI24 solo hay incidencia a partir de la década de los 90. Por último, en el SPI48 la incidencia es desigual, y mientras en Antequera y Málaga son más frecuentes en los años 80 y en la última década, en Algarrobo y Periana lo son en las de los años 80 y 90.

f) El número de meses afectados por sequías hidrológicas severas es menor, con un patrón de distribución espacio-temporal similar al anterior, pudiendo identificarse a partir de los años 80. Con diferentes matices, en las décadas de los 80 y 90 se produjeron los mayores índices, ya sean para el SPI24 como para el SPI48.

g) Aún más significativo resulta el análisis y distribución del número de meses afectados por sequías hidrológicas extremas, con una especial incidencia en todos los observatorios en la década de los 90, como encuentran VICENTE-SERRANO et al., (2004) en valencia y VICENTE-SERRANO, (2006a y b) en el mediterráneo español.

En definitiva, las sequías hidrológicas han tenido importancia a partir de la década de los años 80, con una mayor duración en los observatorios de las cabeceras de cuenca y una mayor intensidad en los orientales. Resulta significativa, la escasa o nula incidencia que la sequia 2004-2006 tuvo en Málaga, en la que las autoridades activaron distintos planes de adaptación a la sequía, mientras que esta, si tuvo una especial incidencia en Antequera, cabecera de la cuenca regulada (CMA, 2007).

\subsection{Percepción social de las sequías desde la agenda massmediática}

El análisis de las rachas secas y sequías hidrológicas en 4 observatorios representativos de la provincia de Málaga ha puesto de manifiesto que estas poseen una distribución irregular en tiempo y espacio. De todas las anomalías secas contabilizadas, solo en 4 ocasiones $(1975,1983,1985,1999)$ tienen una representación generalizada en el área de estudio. En 6, solo afecto a 3 observatorios, en 13 a 2, y en 12 a uno solo. 
Pero además, a las características clásicas de las sequias mediterráneas ya reseñadas por diversos autores para el litoral español, (MAHERAS et al., 1999; VICENTE-SERRANo et al., 2004; Vicente-Serrano y LóPez-Moreno, 2005; Cuadrat Prats y Vicente-SERRANO, 2005; PoQuet et al., 2008), hemos de añadir un marcado cambio de tendencia a partir de la década de los 80 .

La evolución acumulada del número de meses secos muestra como hasta iniciados los años 80, existe una tendencia lineal o cuasi-lineal, sin embargo, esta se modifica sustancialmente a partir de estos años, siendo los observatorios del interior, aquellos en los que la ruptura de tendencia adquiere una mayor significación estadística.

Como se puede observar en Fig. 3, donde se establece la correlación entre la evolución de la sequía y la respuesta mediática, cabe constatar que no existe un divorcio significativo entre la realidad y la construcción informativa, aunque la evolución refleja que es durante las dos últimas décadas cuando se ha corregido la debilidad notoria de la actitud del periódico para equiparar la intensidad periodística a la dimensión real del fenómeno y su impacto en la sociedad.

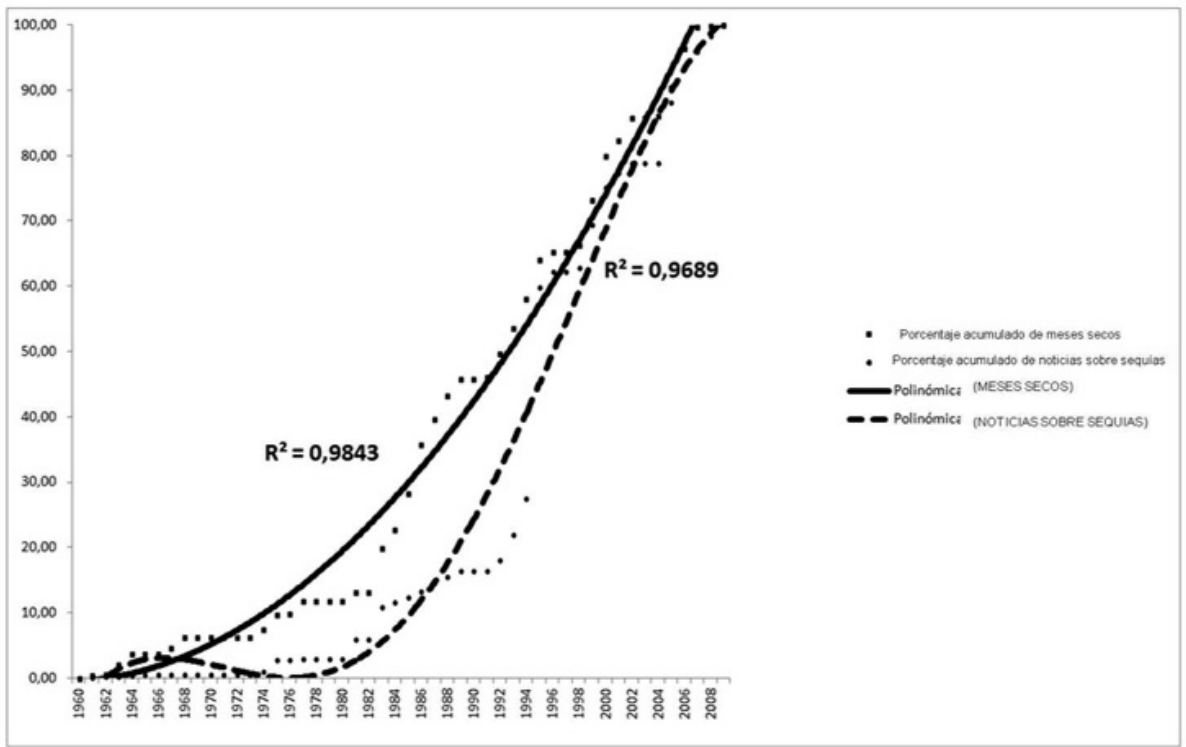

Fig. 3. Evolución acumulada de meses secos y cobertura periodística sobre sequías. (19612009)

El análisis de contenido de los medios de comunicación permite observar de qué modo la percepción social es sustancialmente coherente con estos indicadores.

Y permite concluir que la transferencia de prominencias a la sociedad en relación a las situaciones de sequía es sustancialmente coherente.

\subsubsection{Volumen}

Los indicadores del número de textos de la cobertura periodística dedicada a las sequías muestran una pauta muy equilibrada con los registros descritos mediante el SPI. 
Las principales sequías calificadas con SPI48 se dan en los años noventa en la totalidad de observatorios evaluados, aunque también se producen puntas severas en el decenio precedente, sobre todo en el interior (Antequera y Periana), así como en la primera década del siglo XXI; y el volumen de espacio dedicado en la prensa se corresponde linealmente con esos datos. De hecho, más de la mitad de los textos publicados en el diario de referencia en el territorio se producen precisamente en los años noventa en coherencia con la gran sequía de estos años.

Así, solo en 1995 se alcanza la cifra de 321 unidades publicadas en el periódico, un promedio considerable en el calendario anual que además representa una tercera parte de la totalidad de unidades en los veintisiete años en los que se ha desarrollado la investigación. Ese año, aun con la sequía en una cota muy acentuada, refleja un comportamiento no obstante infrecuente porque la cifra en 1994 se había elevado a 54 y en 1996 lo haría a 23. Sólo diez años más tarde, con 91 ítems en 2005, vuelve a apreciarse una punta excepcional.

Cuadro 1. Evolución de informaciones periodísticas relativas a las sequías (1960-2009).

\begin{tabular}{|c|c|c|c|c|c|c|}
\hline \multirow{2}{*}{ Total } & 1960 & 1970 & 1980 & 1990 & 2000 & Total \\
\hline & 6 & 23 & 133 & 526 & 264 & 952 \\
\hline $\begin{array}{c}\text { Doble } \\
\text { pág }\end{array}$ & - & - & 4,41 & 20,59 & 75 & 68 \\
\hline Página & - & 6,45 & 19,35 & 45,16 & 29,03 & 62 \\
\hline $3 / 4$ & - & 1,35 & 8,07 & $\mid 65,02$ & 25,56 & 223 \\
\hline $1 / 2$ & 1,39 & 2,78 & 11,11 & 55,09 & 29,63 & 216 \\
\hline $1 / 4$ & 0,48 & 1,9 & 20,48 & $\mid 53,81$ & 23,33 & 210 \\
\hline $1 / 8$ & & 2,21 & 15,44 & $\mid 66,91$ & 15,44 & 136 \\
\hline Breve & 5,41 & 8,11 & 32,43 & 43,24 & 10,81 & 37 \\
\hline
\end{tabular}

En los periodos de escasa incidencia, como los años sesenta, el volumen de textos periodísticos no supone siquiera el 1\%, y conjuntamente con el trabajo de campo aplicado a los años setenta se quedan por debajo del 3\%. Desde luego la sequía de los ochenta, con la irrupción de una fuerte cobertura periodística que ya frisa el 14\%, introduce una capacidad de respuesta informativa coherente con la realidad y además lleva esta especialización temática a la redacción del periódico generando unos mecanismos de respuesta más intensos que ya se aprecian en los años noventa y primeros años del siglo XXI, donde se concentran cuatro de cada cinco textos recogidos en el período de los años secos.

Desde 1992, solo en tres años se certifican menos de veinte unidades publicadas acerca de la sequía; hasta ese momento, sólo se había superado esa cota en dos de los catorce años evaluados.

\subsubsection{Tamaño}

Esta variable sondea no ya la amplitud de la cobertura deducida del número de textos periodísticos sino la profundidad de la cobertura según la extensión de éstos, y revela que la atención a la agenda de la sequía supera notoriamente las coberturas periodísticas básicas. Dos tercios de los textos localizados en el trabajo de campo oscilan entre 
$1 / 4$ de página y $3 / 4$ de página. Los textos básicos de $1 / 8$ de página o los breves de menos de cien palabras apenas representan un volumen superior a los grandes trabajos de página completa o doble página. Aunque en la década de los sesenta y setenta, la dimensión del diario se mantenía en parámetros considerablemente más reducidos que en décadas posteriores, resulta patente que entonces no abundaban los trabajos de gran relieve que comienzan a generalizarse a partir de los años ochenta.

Esto es coherente con la irrupción de grandes sequías, pero también con un compromiso periodístico cada vez más dinámico. En los años 1970s, concretamente 1974 y 1975, aparecen los primeros trabajos a página completa y doble página, aunque todavía los textos de tamaño menor representan su proyección más significativa. En la década de 1980s, el balance es desigual, ya que en 1981 o 1983 coinciden coberturas de gran tamaño con soluciones cortas, pero, en la segunda mitad del decenio, el estándar de tamaño se sitúa entre $1 / 4 \mathrm{y} 3 / 4$ de página. En los años noventa, los grandes textos ya representan un $13 \%$, pero en la primera del siglo XXI sólo los trabajos de doble página ya son uno de cada cinco, frisando un $20 \%$ muy significativo. Y además durante esta década se consolida una variedad en las coberturas que proyecta el tratamiento periodístico en todas las modalidades, con una mayor voluntad de equilibrio.

\subsubsection{Género}

Este indicador permite sondear la complejidad del tratamiento periodístico más allá de su amplitud y profundidad, en función de la tipología de textos desde las modalidades básicas de la noticia -limitada a la descripción enunciativa de unos pocos datos sobre un determinado hecho casi siempre atomizado- hasta el reportaje en profundidad (deep reporting) o los artículos de análisis con reflexiones deliberativas; y los resultados una vez más apuntan en la dirección de los datos ya evacuados en los campos anteriores.

Cuadro 2.- Género de informaciones periodísticas relativas a las sequías

\begin{tabular}{|c|c|c|c|c|c|c|}
\hline Década & $\mathbf{1 9 6 0}$ & $\mathbf{1 9 7 0}$ & $\mathbf{1 9 8 0}$ & $\mathbf{1 9 9 0}$ & $\mathbf{2 0 0 0}$ & Total \\
\hline Total & 6 & 23 & 133 & 526 & 264 & $\mathbf{9 5 2}$ \\
\hline GÉNERO & \multicolumn{7}{|c|}{} & \\
\hline Noticia & 0,29 & 1,43 & 14,06 & 59,68 & 25,53 & 697 \\
Reportaje & 1,07 & 1,07 & 13,9 & 42,25 & 41,71 & 187 \\
Editorial & - & 25 & 12,5 & 50 & 12,5 & 8 \\
Artículo & 5,71 & 14,29 & 5,71 & 62,86 & 11,43 & 35 \\
Entrevista & - & 16 & 24 & 20 & 40 & 25 \\
\hline
\end{tabular}

Hay que anotar que tres cuartas partes de los textos se atribuyen a la denominación genérica de 'noticia' como texto informativo, una categoría que integra una amplia diversidad de modalidades según su tamaño, desde el breve de pocas líneas al reportaje o incluso el informe documental. Se trata de un volumen elevado respecto a los textos interpretativos o de opinión, pero en la lógica de los medios. Hay varios aspectos que conviene anotar: con la irrupción de coberturas de amplio espectro sobre la sequía en los años ochenta, aparece el reportaje hasta representar ya un interesante $15 \%$. Hasta entonces, en los 60's y 70's, destacaba apenas la presencia de tribunas de opinión como proyección de especialistas naturalmente poco críticos; pero es en los 80 's cuando la prensa emite señales de estar en condiciones de dar una respuesta de mayor cualifica- 
ción. En los 90's, además de ese 15\% de reportajes, se constata asimismo una creciente presencia en la sección editorial, que cualifica el tema como ítem con centralidad para el medio, y sobre todo artículos firmados. En los primeros años del siglo XXI, el reportaje aún crece al $20 \%$ y la entrevista adquiere una proyección significativa como procedimiento de sondear voces especializadas bajo la dirección del periodista.

De hecho, entre 2001 y 2007, la cobertura mediante reportajes y crónicas oscila siempre entre el 20 y el 35 por ciento, un valor sostenido que descarta cualquier interpretación circunstancial. De hecho, excepto textos de opinión, se observa que lo característico es utilizar el conjunto de modalidades periodísticas propiciando coberturas más ricas y elaboradas. Así, mientras entre 1992 y 1996 las noticias aún representaban entre el 70 y el 85 por ciento; a finales de los noventa ya desciende para situarse en torno al 605 de manera regular, por la mayor aplicación a través de otros géneros.

\subsubsection{Fuentes}

El campo de las fuentes constituye una referencia clave para el análisis de las coberturas periodísticas ya que revela la categoría del trabajo en el doble eje de canti$\mathrm{dad} /$ calidad de las voces de referencia: a mayor presencia de fuentes, mayor diversidad dialéctica; a mayor calidad de las fuentes, un resultado más cualificado. Y este campo sí delata una debilidad apreciable, aunque hay que apuntar que se trata de una debilidad estructural del periodismo español que se proyecta también al tema de las sequías.

Cuadro 3.- Fuentes de las informaciones periodísticas relativas a las sequías

\begin{tabular}{|c|c|c|c|c|c|c|}
\hline Década & $\mathbf{1 9 6 0}$ & $\mathbf{1 9 7 0}$ & $\mathbf{1 9 8 0}$ & $\mathbf{1 9 9 0}$ & $\mathbf{2 0 0 0}$ & Total \\
\hline & 6 & 23 & 133 & 526 & 264 & $\mathbf{9 5 2}$ \\
\hline Fuentes & \multicolumn{7}{|l|}{} & \\
\hline Documental & - & 3,23 & 19,35 & 54,84 & 22,58 & 62 \\
Personal & 3,01 & 8,43 & 15,66 & 56,02 & 16,87 & 166 \\
Especializada & - & - & 11,67 & 66,67 & 21,67 & 180 \\
Institucional & 0,15 & 1,04 & 11,79 & 56,42 & 30,6 & 670 \\
Testimonial & - & 4 & 16 & 60 & 20 & 25 \\
\hline
\end{tabular}

-Sobre la cantidad: Los textos de los 60's y 70's oscilan en el promedio de fuente única, donde no se trata de contrastar o confirmar nada, sino de proyectar una voz, casi siempre oficial. Pero este registro previsible se conserva en buena medida en los 80 's, ya que, a pesar de catapultarse el volumen de las coberturas, no ocurre así con las fuentes que apenas suponen 1.15 por texto, nivel que no varía sustancialmente en los 90's, con 1.2 por texto, o en la década siguiente, 1.1.

-Sobre la calidad: en los años 80 's aparece la fuente especializada, clave para asegurar que la cobertura adquiera cualificación competente, y se sitúa en el 15\% del total; un nivel que se eleva en los 90 's hasta el $20 \%$ aunque retrocede después al $15 \%$ nuevamente en los primeros años del siglo XXI. Se trata de un nivel significativo pero notoriamente solapado por la dimensión de las fuentes institucionales, que representan el altavoz dominante para dar respuesta sobre la sequía: en los ochenta frisa el $60 \%$, y ha seguido creciendo hasta pasar del $70 \%$ en los 90 's y aproximarse después al $80 \%$. Se trata de una evolución inquietante ya que retrata un tratamiento periodístico cada vez más alejado de la realidad social. La reducción de fuentes especializadas puede relacionarse con la tendencia a abaratar el trabajo periodístico, con una 
reducción de capital intelectual que se manifiesta en la profesionalidad y sus resultados prácticos; así, no se han repetido cifras de fuentes especializadas de hasta el 55\% en 1986 respecto a las sequías, o el $40 \%$ en 1989, y aún en torno al $33 \%$ en 1992 1995 , con excepciones puntuales en 1999 y 2000 . Ni siquiera los testimonios y voces personales, que suben en otros temas como reflejo de un periodismo más popular, resultan aquí apreciables. Y este carácter institucional, que alcanza cotas en la proyección de las fuentes del $85 \%$ en 2005 o el $84 \%$ en 2007 , es además un factor de disuasión del interés del lector (DíAZ NosTY, 1996) a contracorriente con los objetivos teóricos de incrementar la sensibilidad social.

\subsubsection{Tono}

Este campo, metodológicamente conflictivo al requerir apreciaciones subjetivas, permite abordar los enfoques de las coberturas sobre las sequías puesto que limitarse a la deducción numérica de la agenda supone ignorar que las noticias transmiten significados acerca de la realidad.

Cuadro 4.- Tono de las coberturas periodísticas sobre las sequías

\begin{tabular}{|c|c|c|c|c|c|c|}
\hline Década & $\mathbf{1 9 6 0}$ & $\mathbf{1 9 7 0}$ & $\mathbf{1 9 8 0}$ & $\mathbf{1 9 9 0}$ & $\mathbf{2 0 0 0}$ & Total \\
\hline Total & 6 & 23 & 133 & 526 & 264 & 952 \\
\hline TONO & & & & & & \\
\hline Dramático & 1,44 & 2,88 & 12,95 & 58,27 & 24,46 & 139 \\
Crítico & - & 0,97 & 3,86 & 74,88 & 20,29 & 207 \\
Analítico & 0,76 & 2,65 & 19,32 & 45,45 & 31,82 & 264 \\
Realista & 0,38 & 1,13 & 15,47 & 53,58 & 29,43 & 265 \\
Desdramatizador & 1,3 & 9,09 & 19,48 & 36,36 & 33,77 & 77 \\
\hline
\end{tabular}

Y además, la existencia de tono se constata al observar que el discurso objetivista, que se corresponde con la jerga característicamente informativa, es inferior al número de noticias desde los años 60's y 70's, y de hecho apenas representa el $40 \%$ del volumen de noticias en los 80 's, menos del $35 \%$ en los 90 's y superando un todavía discreto $45 \%$ en la década posterior. Al haber más textos informativos que discursos de tono informativo, se deduce la 'intencionalidad' en el tratamiento de la sequía. No obstante, se constata que lo predominante es complementar la información con enfoques explicativos, entre la pedagogía y el tono de análisis, ya relevante en la dictadura franquista y notorio desde los 80 's, cuando alcanza un tercio del volumen, aunque luego desciende al $23 \%$ pero en los primeros años del siglo XXI recupera un $29 \%$. En concreto, en los años ochenta se alcanzan cotas significativas ene se tratamiento analítico hasta el $62 \%$ en 1988, el 55\% en 1986 o el 46,9\% en 1983 . Son indicadores muy considerables que no se repiten posteriormente, ya que en los noventa solo en 1993 y 1996 se sitúan los textos analíticos en torno al 40\%, y los restantes años entre el $10 / 25 \%$; pero en los últimos, entre 2005 y 2007 , vuelve a alcanzar niveles del $40 \%$. Por el contrario, el tono dramático se contiene en porcentajes discretos del $13 \%$ al $15 \%$ en las últimas décadas; y de hecho el tono de 'desdramatización' en los 80 's y siglo XXI es similar al 'dramático', no así en los 90's aunque entonces la sequía alcanzaba sus cotas de mayor dureza, momento en el que aparece de forma natural un porcentaje acentuado de textos con tono 'crítico hasta el 30\%, doblando el nivel de las décadas anterior y posterior. 


\subsubsection{Temas}

La agenda temática en torno a la sequía a lo largo de estas cinco décadas está encabezada por varias líneas preponderantes: 'medidas adoptadas por la Administración' (105 ítems), 'recursos hídricos' (101), 'daños en el sector agrícola' (90), ‘abastecimiento humano' (89), a las que siguen 'climatología' (80) e ‘infraestructuras hidráulicas' (79). Estos argumentos copan el mayor número de referencias, como puede constatarse en el cuadro 5, sobre otros de incidencia media-alta como 'restricciones [en el abastecimiento]' (65), 'captaciones, sondeos y prospecciones' (56), 'abastecimiento humano' (53) y 'conflictos entre administraciones' (51). En definitiva se pueden establecer asociaciones para agrupar categorías dominantes: 'recursos, abastecimiento y restricciones' $(101+89+53)$, 'reacciones de la Administración' $(105+51)$, 'infraestructuras y prospecciones' $(79+56)$, y en menor medida 'efectos en la agricultura' y 'climatología'.

Cuadro 5.- Evolución temática de la sequía por décadas

\begin{tabular}{|c|c|c|c|c|c|c|c|c|}
\hline & \multicolumn{7}{|c|}{ Sequías } & \multirow[b]{2}{*}{ Total } \\
\hline & 1966-8 & $74-77$ & 1981 & $1983-9$ & 1992-6 & 1998-02 & 2004-7 & \\
\hline Noticias & 6 & 23 & 30 & 103 & 493 & 165 & 156 & 976 \\
\hline Meses secos & 26 & 16 & 8 & $\$ 2$ & 48 & 49 & 38 & 267 \\
\hline $\begin{array}{c}\text { Influencia sobre el } \\
\text { turismo }\end{array}$ & & & & & 15 & & 0 & 15 \\
\hline Daño a la ganaderia & & 1 & 1 & 1 & 0 & & 0 & 3 \\
\hline Sequía ciclica & & 1 & & & 0 & & 0 & 1 \\
\hline $\begin{array}{c}\text { Daño a agricultura } \\
\text { Abastecimiento }\end{array}$ & 4 & 5 & 11 & 7 & 37 & 15 & 11 & 90 \\
\hline $\begin{array}{l}\text { humano } \\
\text { Conflicto entre }\end{array}$ & 1 & 3 & & 16 & 40 & 17 & 12 & 89 \\
\hline $\begin{array}{l}\text { administraciones } \\
\text { Intervención de la } \\
\text { Iglesia }\end{array}$ & & & & $\begin{array}{l}1 \\
1\end{array}$ & $\begin{array}{c}31 \\
0\end{array}$ & 16 & $\begin{array}{l}3 \\
0\end{array}$ & $\begin{array}{l}51 \\
1\end{array}$ \\
\hline $\begin{array}{l}\text { Recursos hidricos } \\
\text { Infraestructuras }\end{array}$ & & 3 & 1 & 25 & 26 & 26 & 20 & 101 \\
\hline $\begin{array}{l}\text { hidraulicas } \\
\text { Trasvases de agua }\end{array}$ & & & & 4 & $\begin{array}{l}38 \\
27\end{array}$ & $\begin{array}{c}22 \\
4\end{array}$ & $\begin{array}{c}15 \\
6\end{array}$ & $\begin{array}{l}79 \\
37\end{array}$ \\
\hline $\begin{array}{l}\text { Efectos en el Clima } \\
\text { Captaciones, } \\
\text { sondeos y }\end{array}$ & 1 & 6 & 3 & 13 & 23 & 14 & 20 & 80 \\
\hline $\begin{array}{c}\text { prospecciones } \\
\text { Campanias de } \\
\text { concienciación / } \\
\text { Consejos de ahorro }\end{array}$ & & 2 & 1 & 5 & 38 & 2 & 15 & $\begin{array}{l}56 \\
32\end{array}$ \\
\hline $\begin{array}{c}\text { Fuente de energia } \\
\text { Préstamos } \\
\text { bancarios/ }\end{array}$ & & & 1 & & 0 & & 0 & 1 \\
\hline $\begin{array}{l}\text { Subvenciones ante } \\
\text { adversidad clims } \\
\text { Medidas de la }\end{array}$ & & & 4 & & 7 & 3 & 2 & 16 \\
\hline $\begin{array}{l}\text { administración } \\
\text { Consecuencias }\end{array}$ & & 1 & 2 & 7 & 51 & 13 & 31 & 105 \\
\hline medioambientales & & & 1 & 4 & 7 & 5 & 1 & 18 \\
\hline
\end{tabular}




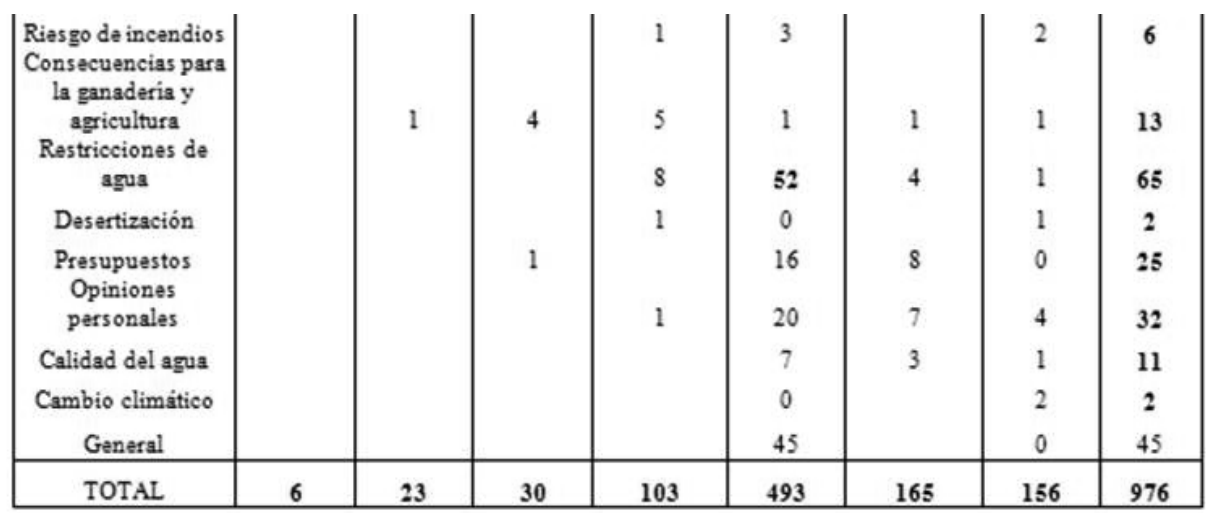

Hay que constatar, al margen de la referencia 'climatología' que remite a las predicciones y a la evolución del comportamiento del clima, la escasa incidencia de cuestiones de alta centralidad como 'desertización' (2 ítems), 'cambio climático' (2) o 'sequía cíclica' (1). Conjuntamente este grupo de temas apenas representan más que un $0,5 \%$ del total. La desertización y el cambio climático son referencias sobre todo de la primera década del siglo XXI, pero incluso en este periodo de muy moderada presencia, toda vez que suponen el 1,9\% de las referencias consignadas en el periodo 2004-2007 con 38 meses secos.

Es interesante anotar la progresiva ampliación de la agenda temática. En las últimas décadas, con el incremento considerable de la respuesta periodística a la cuestión genérica de la sequía, se constata una variedad creciente de orientaciones específicas, enfoques y planteamientos que definen une percepción más compleja del carácter sistémico del fenómeno, que en los sesenta y setenta se limitaba a interpretaciones más planas, apenas significativa por los efectos sobre la agricultura.

Lejos de los datos de los años sesenta y setenta, incluso en los primeros años de la década posterior, cuando la escasa incidencia informativa coincidía con una débil pluralidad de enfoques, a partir de la segunda mitad de los ochenta, en ese largo periodo de 82 meses secos, afloran nuevas orientaciones en la agenda con un relieve ya significativo: infraestructuras hidráulicas, abastecimiento humano o climatología, que suman 54 ítems, más de la mitad de las referencias del periodo. No obstante, aún se trata de enfoques elementales que remiten a cuestiones de servicio de abastecimiento, garantías de éste y prospectiva. Sólo en los años noventa, con el desarrollo exponencial de la cobertura periodística, la agenda se reorienta para incorporar planteamientos económicos y sociales de recorrido ensanchado, como los trasvases o los efectos en el turismo, principal industria del territorio.

Hay, no obstante, una focalización definida de las grandes líneas argumentales, que en la primera década del siglo XXI de nuevo acaparan mayor protagonismo hasta aglutinar el $64,7 \%$ de la agenda en sólo cinco etiquetas: medidas de la administración, climatología, recursos hídricos, infraestructuras hidráulicas y campañas de concienciación. Estas últimas, con 15 ítems, representan en este período el 9,6\% del total, un volumen apreciable en contraste con lo ocurrido en las grandes sequías de los años noventa con apenas 9 y 5 ítems respectivamente, que suponen el 1,8 y 3 por ciento en los ciclos 1992-1996 y 1998-2002. 


\section{Conclusiones}

1. No hay una distorsión de la percepción social de la sequía a través de los medios. La tensión de la cobertura periodística revela, de hecho, un alto grado de coherencia con los datos registrados por el Standarized Precipitation Index (SPI). El volumen de la información es muy bajo en los años sesenta y setenta, a pesar de su relevancia en el discurso político de la época; y emerge con relieve en la agenda de la prensa a partir de las sequías reales de los ochenta y, sobre todo, los noventa, cuando alcanza su máxima intensidad.

2. El uso del SPI es una herramienta útil para el análisis tipológico de las anomalías secas, especialmente en series temporales amplias. Así se aprecia una frecuente aparición de rachas secas extremas, sobre todo durante las décadas de los años 80 y 90. En la última década, se contabilizan algunos episodios en las cabeceras y litoral oriental, sin que este tipo de alteraciones afecte al observatorio de Málaga, el área de una mayor aglomeración demográfica.

3. Las sequías hidrológicas han sido menos frecuentes y de peores consecuencias desde el punto de vista de las reservas hídricas. Han tenido importancia a partir de la década de los años 80, con una mayor duración en los observatorios de las cabeceras de cuenca y una mayor intensidad en los orientales. Resulta significativa, la escasa o nula incidencia que la sequia 2004-2006 tuvo en Málaga, mientras que si tuvo una especial incidencia en Antequera, cabecera de la cuenca regulada.

4. Durante las últimas décadas del siglo XX, coincidiendo con sequías relevantes, se consolida en los medios una especialización temática para dar respuesta periodística a esa realidad. Esto constata un interés objetivo que modula la percepción social con mensajes cada vez más elaborados. En la última década del siglo XX, los grandes textos ya son casi el 15\%; y alcanzan el $20 \%$ en los primeros del siglo XXI. Se trata de géneros cada vez más exigentes, sobre todo el reportaje.

5. Las grandes áreas temáticas proyectadas a la opinión pública no carecen de lógica pública incluso secuencial: recursos, abastecimiento y restricciones; reacciones de la Administración; e infraestructuras y prospecciones. La agenda-setting se amplía tras las grandes sequías de la última década del siglo XX con mayor complejidad de asuntos socioeconómicos; pero sólo en el siglo XXI empieza a ser significativa la etiqueta 'campañas de concienciación', frisando el diez por ciento de los ítems, mientras en la década anterior su franja era 1,5-3\%.

\section{Referencias bibliográficas}

BHALME, H. Nicholas \& MOOLEY, D. Albert (1980): "Large-scale drought/floods and monsoon circulation". Monthly Weather Review 108: 1197-1211.

BARDIN, Laurence (1996): El análisis de contenido. Madrid, Akal.

Blanco CASTILla, Elena (2005): Información local y franquismo. El caso de Diario Sur (1937-1975). Málaga, I+D.COM.

BOYKOFF, Maxwell (2008): "Media and Scientific Communication: A case of Climate Change". Geological Society, Special Publication 2008, v305, pp. 11-18.

BORDI, Isabella; FRIGIO, Sandro; PARENTI, Patriaia; SPERANZA, Antonio; \& SUTERA, Alfonso (2001): "The analysis of the Standardized Precipitation Index 
in the Mediterranean area: Large-scale patterns". Annals of Geophysics 44 (5-6), pp. $965-978$

BORDI, Isabella; FRIGIO, Sandro; PARENTI, Patriaia; SPERANZA, Antonio; SUTERA, Alfonso (2001): The analysis of the Standardized Precipitation Index in the Mediterranean area: Regional patterns. Annals of Geophysics 44 (5-6), pp. 979-993.

CHANGNON, Stanley \& EASTERLING, Williams (1989): "Measuring drought impacts: the Illinois case". Water Resources Bulletin, 25, pp. 27-42.

CMA (Cuenca Mediterránea Andaluza) (2007): Plan especial de actuación en situaciones de alerta y eventual sequía en la cuenca mediterránea andaluza. Consejería de Medio Ambiente. Junta de Andalucía. 233 pp.

COLEMAN, Stephen; ANTHONY, Scott \& MORRISON, David (2009): Public Trust In the News. A constructivist study of the social life of the news. Oxford, Reuters Institute for the Study of Journalism.

EASTERLING, David (2000): "Climate extremes: observations, modelling and impacts". Science, 289, pp. 2068-2074.

EDWARDS, Daniel \& MCKEE, Beth (1997): "Characteristics of $20^{\text {th }}$ century drought in the United States at multiple time scales". Atmospheric Science Paper No. 634.

EDWARDS, Daniel (2001): Methodology of SPI. http://ccc.atmos.colostate.edu /SPI.htm

ENTMAN, Robert M. (1993): "Framing: toward clarification of a fractured paradigm". Journal of Communication. 434:51-58.

GUTTMAN, Nathaniel (1998): "Comparing the Palmer Drought Index and the Standardized Precipitation Index". Journal of American Water Resources Association. 34 (1): 113-121.

GUTTMAN, Nathaniel (1999): “Accepting the Standardized Precipitation Index: a calculation algorithm". Journal of American Water Resources Association. 35 (2): 311-322.

HAYES, Mathieu (1999): "Monitoring the 1996 drought using the Standardized Precipitation Index". Bulletin of the American Meteorological Society, 80, pp. 429-438.

HOUGHTON, John (2001): Climate Change 2001: The scientific Basis. Cambridge, Cambridge University Press.

IGARTUA, Juan José (2006): Métodos cuantitativos de investigación en comunicación. Barcelona, Bosch.

KEYANTASH, John \& DRACUP, John (2002): “The quantification of drought: an evaluation of drought indices". Bulletin of the American Meteorological Society, 83, pp. 1167-1180.

KOMUSCU, Alí (1999): "Using the SPI to analyze spatial and temporal patterns of drought in Turkey". Drought Network News, 11, pp. 7-13.

KRIPPENDORFF, Klaus (2002): Metodología de análisis de contenido: Teoría práctica. Barcelona, Paidós. 
KRIPPENDORFF, Klaus \& BOCK, Mary Angela (editors, 2008): The Content Analysis Reader. Thousand Oaks, CA: Sage Publications.

KUNKEL, Kenneth. E.; PIELKE, Roger \& CHANGNON, Stanley (1999): “Temporal fluctuations in weather and climate extremes that cause economic and human health impacts: a review". Bulletin of the American Meteorological Society, 80, pp. 1077-1098.

LADLE, Richard et al (2004): "Crying wolf over risks of extinction". Nature, 428:729.

LANA, Xavier; SERRA, Cesar; y BURGUEÑO, Augusto (2001): "Patterns of monthly rainfall shortage and excess in terms of the standardized precipitation index for Catalonia (NE Spain)”. International Journal of Climatology 21 (13), pp. 1669-1691

LANA, Xavier; BURGUEÑO, Augusto; MARTÍNEZ, María; y SERRA, Cesar (2009): "Una revisión de los análisis estadísticos de las precipitaciones diarias y mensuales en Cataluña". Revista del tiempo y el clima del Mediterráneo occidental. Tethys, 6, 15-30,

LLOYD-HUGHES, Benjamin \& SAUNDERS, Mark (2002): "A drought climatology for Europe”. International Journal of Climatology, 22, 1571-1592.

LUHMANN, Niklas (1996): Die Realität der Massenmedien. Westdeutscher Verlag, Opladen.

MAHERAS, Steve; XOPLAKI, Elena \& KUTIEL, Haim (1999): "Wet and dry monthly anomalies across the Mediterranean basin and their relationship with circulation, 1860-1990". Theoretical and Applied Climatology 64 (3-4), pp. 189-198

MARTÍNEZ-MURILLO, J. Francisco y RUIZ SINOGA, J. Damián (2007): “Seasonal changes in the hydrological and erosional response of a hillslope under dryMediterranean climatic conditions (Montes de Málaga, South of Spain)". Geomorphology 88: 69-83.

MCCOMBS, Maxwell (1992): "Explorers and surveyors: expanding strategies for agenda-setting research". Journalism Quaterly 69, pp.813-824.

MCKEE, Thomas; DOESKEN, Nolan \& KLEIST, John (1993): "The relationship of drought frequency and duration to time scales. Eight Conf". Applied Climatology, Dallas, TX, Amer. Meteor. Soc. 179-184.

MCKEE, Thomas; DOESKEN, Nolan, \& KLEIST, John (1995): “Drought monitoring in multiple time scales. Ninth. Conf". Applied Climatology, Dallas, TX, Amer. Meteor. Soc. 233-236.

MCKEE, Thomas; DOESKEN, Nolan; KLEIST, John \& SHRIER, Catherine (2000): "A history of drought in Colorado". http://cwrri.colostate.edu. 20 pp.

MORALES, Alfredo; OLCINA, Jorge; y RICO, Maria (2000): "Diferentes percepciones de la sequía en España: adaptación, catastrofismo e intentos de corrección". Investigaciones Geográficas, 23, pp. 5-46. 
MOREIRA, Elsa .E.; COELHO, Carlos A.; PAULO, A.A.; PEREIRA, Luis S. \& MEXIA, Joao T. (2008): "SPI-based drought category prediction using loglinear models". Journal of Hydrology 354 (1-4), pp. 116-130

NELKIN, Dorothy (1987): Selling science: How the press covers science and technology. New York, W.H. Freeman ed,.

NEUENDORF, Kimberly A. (2002): The content analysis guidebook. Thousand Oaks, CA., Sage.

PALMER, Wayne (1965): "Meteorological droughts". U.S. Department of commerce Weather Bureau Research Paper 45, 58 pp.

PALMER, Wayne (1965): “Analyzing spatial patterns of meteorological drought using standardized precipitation index". Meteorological Applications 14 (4), pp. 329336

PEREZ CUEVA, Alejandro y ESCRIVÁ ORTEGA, José (1982): “Análisis de la sequía en el ámbito mediterráneo". Cuadernos de Geografia 30: 1-12.

PETERS, Hans Peter \& HEINRICHS, Harald (2005): Öffentliche Kommunikation über Klimawandel und Sturmflutrisiken. Bedetungskonstruktion durch Experten, Journalisten und Bürger. Jülich, Forschungszentrum Jülich,.

PIÑUEL, José Luis (2002): "Epistemología, metodología y técnicas del Análisis de Contenido". Estudios de Sociolingüistica 3(1), 1-42

POQUET, David; BELDA, Fernando y GARCÍA-HARO, F. Javier (2008a): "Seguimiento y regionalización de la sequía en la Península Ibérica mediante SPI y técnicas de teledetección". En: HERNÁNDEZ, L. y PARREÑO, J. M. (Eds.): Tecnologías de la Información Geográfica para el Desarrollo Territorial. Las Palmas de Gran Canaria, Servicio de Publicaciones y Difusión Científica de la ULPGC.. Pp. 707-713. ISBN: 978-84-96971-53-0.

POQUET, David; BELDA, Fernando y GARCÍA-HARO, F. Javier (2008b): “Regionalización de la sequía en la Península Ibérica desde 1950 hasta 2007 a partir de SPI y una modelización digital del terreno". XXX Jornadas Científicas de la Asociación Española de Meteorología.

RIFFE, Daniel; LACY, Stephen \& FICO, Frederik G. (2005): Analyzing media messages: Using quantitative content analysis in research. $2^{\mathrm{a}}$ ed. Mahwah, Lawrence Erlbaum.

ROOY. M.P. van (1965): “A rainfall anomaly index independent of time and space”. Notos 14: 43.

RUIZ-SINOGA, José Damián y MARTÍNEZ-MURILLO J. Francisco (2009): “Ecogeomorphological system response variability to the 2004-06 drought along a climatic gradient of the Littoral Betic Range (southernSpain)". Geomorphology 103: 351-362.

RUIZ-SINOGA, José Damián; GARCÍA MARÍN, Ramón; MARTÍNEZ MURILLO, J. Francisco; y GABARRÓN GALEOTE, M. Ángel (2010): "Precipitation Dy- 
namics in southern Spain: trends and cycles". Internacional Journal of Climatology, 30. DOI: 10.1002/joc.2235

SÁNCHEZ ARANDA, José Javier (2005): “Análisis de contenido cuantitativo de medios". En BERGANZA CONDE, María Rosa y RUIZ SAN ROMÁN, José Antonio (coords.): Investigar en Comunicación. Madrid, McGraw Hill.

SIRDAŞ, Sevinç \& ŞEN, Zekai (2001): "Application of the standardized precipitation index (SPI) to the Marmara region, Turkey". IAHS-AISH Publication (272), pp. 291-296

SHUKLA, Shraddhanand \& WOOD, Andrew (2008): "Use of a standardized runoff index for characterizing hydrologic drought". Geophysical Research Letters 35 (2), art. no. L02405.

THORNTHWAITE, Carles W. (1948): “An approach toward a rational classification of climate". Geographical Review 38: 55-94.

VICENTE-SERRANO, Sergio M.; GONZÁLEZ-HIDALGo José C.; DE LUIS, Miguel; y RAVENTÓS, José (2004): "Drought patterns in the Mediterranean area: The Valencia region (eastern Spain)". Climate Research 26 (1), pp. 5-15.

VICENTE-SERRANO, Sergio M. y LOPEZ-MORENO, Juan (2005): “Hydrological response to different time scales of climatological drought: An evaluation of the Standardized Precipitation Index in a mountainous Mediterranean basin". Hydrology and Earth System Sciences 9 (5), pp. 523-533

VICENTE-SERRANO, Sergio M. (2006a): "Differences in spatial patterns of drought on different time scales: An analysis of the Iberian Peninsula". Water Resources Management 20 (1), pp. 37-60

VICENTE-SERRANO, Sergio M. (2006b): "Spatial and temporal analysis of droughts in the Iberian Peninsula (1910-2000)". Hydrological Sciences Journal 51 (1), pp. 83-97

WILHITE, Donald A. (1993): Drought assessment, management and planning: Theory and case studies. Boston, Kluwer.

WIMMER, Roger D. y DOMINICK Joseph R. (2005): Mass Media Research: An Introduction. $8^{\text {a }}$ ed. Belmont, CA., Wadsworth.

WU, Hong; HAYES, Michael; WEISS, Albert; \& HU, Qi (2001): "An evaluation of the standardized precipitation index, the china-z index and the statistical z-score". International Journal of Climatology 21: 745-758. 